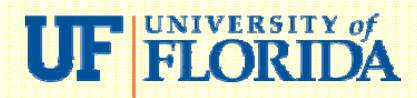

IFAS Extension

ENY-854

\section{Quick Reference Guide to Citrus Insecticides and Miticides}

M.E. Rogers, P. A. Stansly, L. L. Stelinski and J. D. Yates

\begin{tabular}{|c|c|c|c|c|c|c|c|c|c|c|c|c|}
\hline \multirow[b]{2}{*}{$\begin{array}{l}\text { Pesticide active } \\
\text { ingredient }\end{array}$} & \multirow[b]{2}{*}{$\begin{array}{l}\text { Product Brand } \\
\text { Name Examples }\end{array}$} & \multirow[b]{2}{*}{$\begin{array}{l}\text { Restricted entry } \\
\text { interval (REI) }\end{array}$} & \multirow[b]{2}{*}{$\begin{array}{c}\text { Pre-harvest } \\
\text { interval } \\
\text { (PHI) }\end{array}$} & \multicolumn{8}{|c|}{ Target pest } & \multirow[b]{2}{*}{$\begin{array}{c}\text { Effects on } \\
\text { natural } \\
\text { enemies }\end{array}$} \\
\hline & & & & $\begin{array}{l}\text { Mode of } \\
\text { Action }^{1}\end{array}$ & Psyllid & Leafminer & $\begin{array}{l}\text { Rust } \\
\text { Mites }\end{array}$ & $\begin{array}{l}\text { Spider } \\
\text { Mites }\end{array}$ & $\begin{array}{c}\text { Root } \\
\text { Weevil } \\
\text { Adults }\end{array}$ & $\begin{array}{c}\text { Scale } \\
\text { Insects }\end{array}$ & Mealybugs & \\
\hline Abamectin + oil & Agri-mek 0.15EC & 12 hours & 7 days & 6 & ++ &,$+++ R$ &,$+++ \mathrm{R}$ & + & + (oil) & $+($ oil) & + (oil) & medium \\
\hline Acetamiprid & Assail 70WP & 12 hours & 7 days & 4 & - &,$+++ R$ & - & - & $?$ & + & ++ & medium \\
\hline Aldicarb & Temik $15 \mathrm{G}$ & 48 hours & $\begin{array}{l}0 ; 30 \text { days } \\
\text { (lemons) }\end{array}$ & $1 \mathrm{~A}$ &,$+++ R$ & - &,$+++ R$ & +++ & - & - & - & low \\
\hline Carbaryl & Sevin XLR Plus & 12 hours & 5 days & $1 \mathrm{~A}$ & ++ & - & + & - &,$+++ R$ &,$+++ R$ & + & high \\
\hline Chlorpyrifos & Lorsban 4E & 5 days & 21 days & $1 \mathrm{~B}$ &,$+++ R$ & + & + & - & + &,$+++ R$ &,$+++ R$ & high \\
\hline Diflubenzuron & Micromite 80WGS & 12 hours & 21 days & 15 & ++ &,$+++ R$ &,$+++ R$ & - &,$+++ R$ & - & - & low \\
\hline Dimethoate & Dimethoate 4E & 48 hours & $15-45$ days & $1 \mathrm{~B}$ & +++ & - & - & - & ? &,$+++ R$ & + & high \\
\hline Fenbutatin oxide & Vendex 50WP & 48 hours & 7 days & 12 & - & - &,$+++ R$ &,$+++ R$ & - & - & - & low \\
\hline Fenpropathrin & Danitol 2.4EC & 24 hours & 1 day & 3 &,$+++ R$ & - & + & + &,$+++ R$ & - & + & high \\
\hline $\begin{array}{l}\text { Imidacloprid } \\
\text { (soil) }\end{array}$ & Admire Pro & 12 hours & 0 & 4 &,$+++ R$ &,$+++ R$ & - & - & + & ++ & + & low \\
\hline $\begin{array}{l}\text { Imidacloprid } \\
\text { (foliar) }\end{array}$ & Provado $1.6 \mathrm{~F}$ & 12 hours & 0 & 4 &,$+++ R$ & + & - & - & - & ++ & + & medium \\
\hline Petroleum oil & numerous & 12 hours & 0 & NR & + &,$++ R$ &,$++ R$ & ++ & +(eggs) &,$++ R$ & + & low \\
\hline Phosmet & Imidan 70W & 24 hours & 7 days & $1 \mathrm{~B}$ &,$+++ R$ & - & + & $?$ &,$+++ R$ & $?$ & $?$ & medium/high \\
\hline Pyridaben & Nexter Miticide & 12 hours & 7 days & 21 & - & $?$ &,$+++ R$ &,$+++ R$ & - & - & - & high \\
\hline Spinosad & Spintor 2SC & 4 hours & 1 day & 5 & - &,$+++ R$ & - & - & - & - & - & low \\
\hline Spinetoram & Delegate WG & 4 hours & 1 day & 5 &,$+++ R$ &,$+++ R$ & - & $?$ & $?$ & $?$ & $?$ & low \\
\hline Spirodiclofen & Envidor 2SC & 12 hours & 7 days & 23 & - & - &,$+++ R$ &,$+++ R$ & $?$ & - & - & low \\
\hline Spirotetramat & Movento 240 SC & 24 hours & 1 day & 23 &,$+++ R$ & $?$ &,$+++ R$ & $?$ & $?$ & +++ & $?$ & low \\
\hline Sulfur & numerous & 12 hours & 0 & NR & - & - &,$+++ R$ & +++ & - & ? & ? & $\begin{array}{l}\text { high (short } \\
\text { term) }\end{array}$ \\
\hline Thiamethoxam & Actara 25 WG & 12 hours & 0 & 4 &,$+++ R$ & + & - & - & - & ++ & + & medium \\
\hline Thiamethoxam & Platinum 75 SG & 12 hours & 0 & 4 &,$+++ R$ &,$+++ R$ & - & - & + & ++ & + & low \\
\hline $\begin{array}{l}\text { Zeta- } \\
\text { cypermethrin }\end{array}$ & $\begin{array}{l}\text { Mustang } \\
\text { Insecticide }\end{array}$ & 12 hours & 1 day & 3 &,$+++ R$ & - & - & $?$ & +++ & $?$ & ? & high \\
\hline
\end{tabular}

${ }^{1}$ Mode of action class for citrus pesticides from the Insecticide Resistance Action Committee;

$N R=$ no resistance potential $(R)=$ product recommended for control of pest in Florida Citrus Pest Management Guide

Revised September 2009
$(+++)=$ good control of pest
$(++)=$ short-term control of pest
$(+)=$ low levels of pest suppression
$(-)=$ no observed control of pest
(?) = insufficient data available 


\section{Products labeled for application at reduced volume either by ground or aerial application}

\begin{tabular}{|c|c|c|c|c|c|c|c|}
\hline \multicolumn{4}{|c|}{ ALWAYS READ AND FOLLOW LABEL INSTRUCTIONS } & \multicolumn{2}{|c|}{ Ground Applications } & \multicolumn{2}{|c|}{ Aerial Applications } \\
\hline Product & EPA Reg. \# & $\begin{array}{c}\text { Restricted entry } \\
\text { interval (REI) }\end{array}$ & $\begin{array}{c}\text { Pre-harvest } \\
\text { interval (PHI) }\end{array}$ & Product Rate / A & $\begin{array}{c}\text { Minimum Spray } \\
\text { Volume / A }\end{array}$ & Product Rate / A & $\begin{array}{c}\text { Minimum Spray } \\
\text { Volume / A }\end{array}$ \\
\hline Agri-mek 0.15 EC & $100-898$ & 12 hours & 7 days & $10-20 \mathrm{fl} \mathrm{oz}$ & $\begin{array}{l}\text { Sufficient } \\
\text { coverage }\end{array}$ & $5-20 \mathrm{fl} \mathrm{oz}^{1}$ & 10 gallons $^{1}$ \\
\hline Danitol 2.4 EC² & $\begin{array}{c}59639-35 \\
\text { SLN FL-090003 }\end{array}$ & 1 day & 1 day & $16-21 \mathrm{fl} \mathrm{oz}$ & 2 gallons & $16-21 \mathrm{fl} \mathrm{oz}$ & 5 gallons \\
\hline Delegate WG & $\begin{array}{c}62719-541 \\
\text { SLN FL-090009 }\end{array}$ & 4 hours & 1 day & $3-6 o z$ & 2 gallons & $3-6$ oz & 10 gallons \\
\hline Dimethoate $4 \mathrm{E}^{4}$ & $34704-207-67760$ & 2 days & $15-45$ days & $0.5-1 \mathrm{pts}$ & 5 gallons & $1-2$ qts & 5 gallons \\
\hline Lorsban 4E & $62719-220$ & 5 days & $21-35$ days & $2-12$ pts & 10 gallons & $2-12$ pts & 2 gallons \\
\hline Malathion 5 & $9779-5$ & 12 hours & 7 days & $1.25-2 \mathrm{pts}$ & 3 gallons & $1.25-2 \mathrm{pts}$ & 1 gallon \\
\hline Micromite 80 WGS & $\begin{array}{c}400-487 \\
\text { SLN FL-090010 }\end{array}$ & 12 hours & 21 days & $6.25 \mathrm{oz}$ & 2 gallons & $6.25 \mathrm{oz}$ & 5 gallons $^{3}$ \\
\hline $\begin{array}{l}\text { Mustang } \\
\text { Insecticide }\end{array}$ & $\begin{array}{c}279-3126 \\
\text { SLN FL-090011 }\end{array}$ & 12 hours & 1 day & $4.3 \mathrm{fl} \mathrm{oz}$ & 2 gallons & $4.3 \mathrm{fl} \mathrm{oz}$ & 10 gallons \\
\hline Sevin XLR & $264-333$ & 12 hours & 5 days & $1.5-3 \mathrm{qts}$ & $\begin{array}{l}\text { Sufficient } \\
\text { coverage }\end{array}$ & 1.5 - 3 qts & 10 gallons \\
\hline \multicolumn{8}{|c|}{$\begin{array}{l}{ }^{1} \text { Aerial applications of Agri-mek } 0.15 E C \text { are only labeled for citrus leafminer control. } \\
{ }^{2} \text { The use of spray adjuvants with Danitol } 2.4 \mathrm{EC} \text { is prohibited by label. } \\
{ }^{3} \text { Aerial applications of Micromite } 80 \text { WGS cannot be made within } 1,000 \text { feet of bodies of water. } \\
{ }^{4} \text { Additional dimethoate products with similar use patterns may be available. }\end{array}$} \\
\hline
\end{tabular}

Additional citrus pest management information can be found in the Florida Citrus Pest Management Guide available online at http://www.crec.ifas.ufl.edu/extension/pest/index.htm

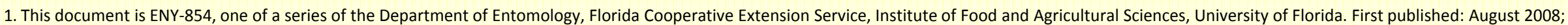
Revised: September 2009.

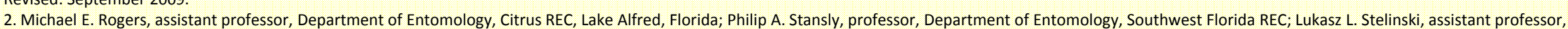

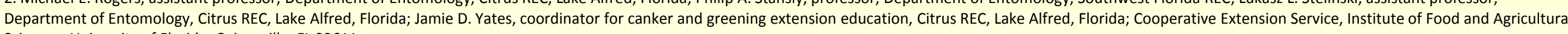
Sciences; University of Florida; Gainesville, FL 32611.

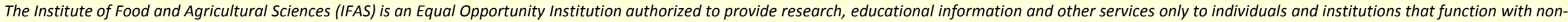

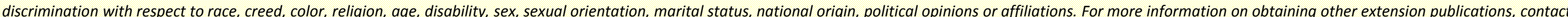

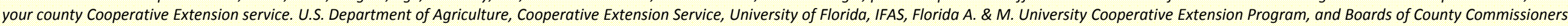
Cooperating. Millie Ferrer, Interim Dean. 